\title{
28 Research Square \\ An Integrative Prognostic and Immune Analysis of PTPRD in Pan-Cancer
}

\section{Chunpei Ou}

longhua district central hospital

Qin Peng

longhua district central hospital

Changchun Zeng ( $\nabla$ zengchch@glmc.edu.cn )

longhua district central hospital, guangdong medical university https://orcid.org/0000-0002-94890627

\section{Research Article}

Keywords: PTPRD, pan-cancer, prognosis, tumor-infiltrating, immunotherapy

Posted Date: June 4th, 2021

DOl: https://doi.org/10.21203/rs.3.rs-569409/v1

License: (c) (i) This work is licensed under a Creative Commons Attribution 4.0 International License. Read Full License 


\section{Abstract}

Background: PTPRD plays an indispensable role in the occurrence of multiple tumors. However, pancancer analysis is unavailable. The purpose of this research was to investigate the relationship between PTPRD and immunity and describe its prognostic landscape across various tumors.

Methods: We explored expression profile, survival analysis, and genomic alterations of PTPRD based on the TIMER, GEPIA, UALCAN, PrognoScan, and cBioPortal database. The frequency of PTPRD mutation and its correlation with response to immunotherapy were evaluated using the cBioPortal database. The relationship between PTPRD and immune-cell infiltration was analyzed by the TIMER and TISIDB databases. A protein interaction network was constructed by the STRING database. GO and KEGG enrichment analysis was executed by the Metascape database.

Results: A significant correlation between PTPRD expression and prognosis was found in various cancers. Aberrant PRPRD expression was closely related to immune infiltration. Importantly, the patients who harbored PTPRD mutation and received immune checkpoint inhibitors had worse overall survival, especially in non-small cell lung cancer and melanoma, and had a higher TMB score. Moreover, PTPRD mutation was involved in numerous biological processes, including immunological signaling pathways. Additionally, a PTPRD protein interaction network was constructed, and genes that interacted with PTPRD were identified. Functional enrichment analysis demonstrated that a variety of GO biological processes and KEGG pathways of PTPRD were primarily involved in the therapeutic mechanisms.

Conclusions: These results revealed that PRPRD might function as a biomarker for prognosis and immune infiltration in cancers, throwing new light on cancer therapeutics.

\section{Introduction}

The prevalence and mortality of cancer are rising worldwide, making cancer the most common cause of mortality in many countries. The American Cancer Society estimates that $1,898,160$ new cancer cases and 608,570 cancer deaths are likely to occur in the United States in 2021 [1]. Therefore, identifying a new target for optimizing cancer treatment is an immediate and severe global demand. The personalized medicine strategies may strengthen the therapeutic benefit by identifying the tumor-specific target or tumor-associated characteristics [2]. Several targeted agents have been developed to improve the efficacy of cancer therapy $[3,4]$. However, not all patients will receive molecular or pathway targeted therapy, which is not generally suitable for all tumor types. Therefore, pan-cancer studies may be valuable for identifying and understanding genes that play a critical role in many cancer types.

Protein tyrosine phosphatase receptor type D (PTPRD) is a member of the protein tyrosine phosphatase (PTP) family, several members of which can regulate a variety of cellular processes, including cell proliferation, differentiation, cell cycle, and malignant transformation [5]. The PTPRD gene is located on chromosome 9p, and its canonical model contains 1912 amino acids, with an estimated mass of 215 $\mathrm{kDa}$. PTPRD is a receptor-type PTP with an extracellular region, which is composed of three Ig-like and 
eight fibronectin type III-like domains, a single transmembrane segment, and two tandem intracytoplasmic catalytic domains, which possess tyrosine-protein phosphatase 1 domain (D1) and tyrosine-protein phosphatase 2 domain (D2). The physiological function of PTPRD depends primarily on its D1 domain, which facilitates the phosphate interaction of cytoplasmic proteins, while the D2 domain can mediate the function of the D1 domain. Recently, the relationship between PTPRD and tumorigenesis has received growing attention. PTPRD participates in multiple signaling pathways, including $\beta$-catenin/ T-cell factor (TCF), signal transduction and activation of transcription 3 (STAT3), extracellular-signalregulated kinase (ERK), and insulin signaling pathway $[6,7]$. The PTPRD expression levels were downregulated in colon cancer, and PTPRD regulated the colon cancer cell adhesion and migration in cooperation with $\beta$-catenin/TCF signaling [8]. Besides, PTPRD deficiency was linked with aggressive behavior in gastric cancer, and PTPRD inactivation facilitated angiogenesis and metastasis of gastric cancer via the upregulation of CXCL8 [9]. These results suggested that PTPRD was involved in the invasion and progression of a variety of tumors. However, PTPRD has not been intensively investigated in the pan-cancer perspective to uncover its expression patterns and the underlying functions and mechanisms in multiple cancers.

Immune checkpoint inhibitors (ICls) have redefined therapies for various types of cancer. However, despite the remarkable performance of ICls, long-lasting therapeutic responses differ among patients [10]. It has recently been found that high microsatellite instability (MSI-H), PD-L1 expression, tumor mutation burden (TMB), gene expression profiles (GEPs), tumor immune microenvironment (TIME), and some specific gene mutations are associated with immunotherapy response [11-13]. Even those biomarkers that have been identified and validated have certain clinical implementation restrictions. For instance, 44-50\% of high TMB or high PD-L1 expression advanced non-small-cell lung cancer (NSCLC) patients had no response to nivolumab plus ipilimumab, while almost $12-15 \%$ of low TMB or low PD-L1 expression patients obtained a partial or complete response in the CheckMate 568 (NCT02659059) study [14]. Therefore, predictive ICls response biomarkers are required to offer appropriate medical assistance to enhance the effectiveness of ICls. Outlining the mechanisms of tumor development and immune infiltration will lay the foundation for future clinical progress. PTPRD restricts STAT3 phosphorylation to suppress the activation of STAT3, contributing to the inhibition of PD-L1 expression, which is involved in the immune response of malignant tumors $[6,15]$. Accordingly, PTPRD has potential predictive significance for immunotherapy [6]. However, the underlying features and mechanisms of PTPRD in pancancer immunology remain unclear.

In this study, it is the first time to conduct a pan-cancer analysis of PTPRD. We intensively investigated PTPRD expression and association with cancer patient prognosis using databases such as TIMER, GEPIA2, UALCAN, PrognoScan. Moreover, the relationship between PTPRD mutation and immunotherapy was further explored using the cBioPortal database, and a survival advantage could be observed in PTPRD mutated patients who obtained ICls. Additionally, we analyzed the association of PTPRD and tumor microenvironments through the TIMER and TISIDB database, indicating that PTPRD is a potential predictive biomarker for immunotherapy. The results illustrated the essential role of PTPRD and provided 
the association and underlying mechanisms between PTPRD and tumor-immune behaviors across multiple cancer types.

\section{Materials And Methods}

\section{Gene expression analysis}

To explore the differential gene expression of PTPRD between tumor and normal tissue, pan-cancer analysis of PTPRD mRNA expression in 32 tumor types from The Cancer Genome Atlas (TCGA) database was performed using the "Diff Exp" module of Tumor Immune Estimation Resource (TIMER; https://cistrome.shinyapps.io/timer/) database [16]. Moreover, the "Expression analysis" module of the Gene Expression Profiling Interactive Analysis (GEPIA2; http://gepia2.cancer-pku.cn/\#analysis) database was used to profile the tissue-wise expression of PTPRD in different cancer types using the data from TCGA and Genotype-Tissue Expression (GTEx) database. The threshold was set according to the following values: P-value cutoff $=0.01$, log2FC (fold change) cutoff $=1$, and "Match TCGA normal and GTEx data". Besides, we profile the expression of PTPRD in different cancer stages using log2 [TPM (Transcripts per million) +1 ] transformed expression data to obtain violin plots. The UALCAN portal (http://ualcan.path.uab.edu/analysis-prot.html) provided the protein expression analysis option for PTPRD using data from the Clinical proteomic tumor analysis consortium (CPTAC) dataset [17].

\section{Survival prognosis analysis}

To investigate the relationship between PTPRD expression and survival across various types of cancers, the survival analysis was performed using the "survival analysis" module of GEPIA2 based on the TCGA and GTEx database. Group cutoff was set as the median. Additionally, the PrognoScan database (http://www.abren.net/PrognoScan/) was used to assess the prognostic value across a wide collection of accessible microarray datasets from the Gene Expression Omnibus (GEO) database. The significance threshold was Cox P-value $<0.05$ and $N>100$ [18].

\section{Genetic alteration analysis}

The cBioPortal (https://www.cbioportal.org/) database was applied to analyze and visualize the genomic data. "TCGA PanCancer Atlas Studies" containing 10,967 samples from 32 studies was selected to query the genetic alteration features of PTPRD, and the "Cancer Types Summary" module exhibited the alteration frequency, mutation type, and cancer types [19]. Additionally, genomic and survival data containing 2,185 samples from four studies were selected to explore the relationship between PTPRD mutation and immunotherapy, and the association of PTPRD mutation with clinical attributes was assessed in the selected cases [20-23].

\section{Immune infiltration analysis}

TIMER database containing 10,897 samples from the TCGA database across 32 cancer types was applied to systematically analyze immune infiltrates with a deconvolution statistical method to deduce 
tumor-infiltrating immune cells (TIICs) abundance from gene expression profiles. We explore the relationship between PTPRD expression and the TIICs abundance, including macrophages, CD4 + T cells, B cells, neutrophils, CD8 + T cells, and dendritic cells. P-values and partial correlation (cor) values were calculated utilizing a purity-adjusted Spearman rank correlation test. Besides, the association between PTPRD mutation and TIICs, including dendritic cells (DCs), B cells, CD $8+T$ cells, M1 macrophages, T cells (general), TAMs, natural killer (NK) cells, T-helper 2 (Th2) cells, follicular helper T (Tfh) cells, M2 macrophages, monocytes, T-helper 1 (Th1) cells, neutrophils, Tregs, T-helper 17 (Th17) cells, and exhausted T cells, were further analyzed using the data from TCGA database [16, 24, 25]. Additionally, the association between PTPRD mutation and infiltrating immune cells was further investigated by TISIDB (http://cis.hku.hk/TISIDB/index.php) database, which integrated data from the TCGA database [26].

\section{Gene correlation analysis}

GEPIA database was applied to obtain genes that had a similar expression pattern with PTPRD expression based on the data from the TCGA database, including 9,736 tumors and 8,587 normal samples, and the top 100 genes were selected based on the correlation coefficient using the Spearman method. Moreover, the correlation analysis was conducted between PTPRD expression and the top 5 gene expression in multiple cancer types and tissues using the Pearson method [27].

\section{Protein-protein interaction (PPI) network analysis}

The STRING (http:// string-db.org) database was applied to exhibit a protein-protein interaction network for PTPRD protein. The hub genes were screened with the following criteria: no more than 50 interactors; low confidence (0.150) as the minimum required interaction score [28].

\section{Functional enrichment analysis}

The systematic and integrative functional enrichment analysis was provided by Metascape (http://metascape.org/gp/index.html\#/main/step1) database. The 100 PTPRD-correlated targeting genes from the gene correlation analysis and the identified 50 PTPRD-correlated targeting proteins from the PPI network analysis were placed into the Metascape database for Gene Ontology (GO) and Kyoto Encyclopedia of Genes and Genomes (KEGG) pathways enrichment analysis [29].

\section{Statistical Analysis}

The Wilcoxon test was applied to assess the expression differences of PTPRD between tumor and normal tissues. Overall survival (OS) was evaluated using Kaplan-Meier method and log-rank tests. The association of PTPRD expression and immune infiltration level was assessed by the Spearman rank correlation test. The correlation of PTPRD mutation and clinical attributes was determined by Kruskal Wallis Test or Chi-squared Test. The $p$-values $<0.05$ were considered statistically significant.

\section{Results}

\section{Gene expression analysis}


To determine the expression differences of PTPRD in tumor tissues and normal tissues, PTPRD mRNA levels in more than 10,000 tumor and normal tissue samples across 23 cancer types from the TCGA database were evaluated using the TIMER database (Supplementary Table 1, Supplementary Table 2). As shown in Fig. 1A, PTPRD was downregulated in lung adenocarcinoma (LUAD), uterine corpus endometrial carcinoma (UCEC), kidney renal clear cell carcinoma (KIRC), bladder carcinoma (BLCA), head and neck carcinoma (HNSC) HPV+, liver hepatocellular carcinoma (LIHC), lung squamous carcinoma (LUSC), glioblastoma multiforme (GBM), thyroid carcinoma (THCA), prostate adenocarcinoma (PRAD), cervical squamous carcinoma (CESC), and stomach adenocarcinoma (STAD) compared with the adjacent normal tissues. Besides, PTPRD was upregulated in head and neck carcinoma (HNSC), and kidney renal papillary cell carcinoma (KIRP) compared to the normal tissues. Moreover, we further explored PTPRD expression across 33 cancer types using the GEPIA2 database, containing the TCGA and GTEx data. As shown in Fig. 1B, PTPRD expression was lower in BLCA, CESC, KIRC, LUAD, LUSC, ovarian serous cystadenocarcinoma (OV), THCA, UCEC, and testicular germ cell tumors (TGCT) compared with the normal tissues, and PTPRD expression was higher in rectum adenocarcinoma (READ) and thymoma (THYM). Moreover, a significant correlation between PTPRD expression and the pathological stages was observed using the GEPIA2 database in multiple cancers, including BLCA, KIRC, and skin cutaneous melanoma (SKCM) (Fig. 1C). These findings suggested that PTPRD might perform diverse functions in cancer progression. Also, the UALCAN database was applied to analyze PTPRD protein expression across tumor and normal cases in the CPTAC datasets, and the results showed that lower protein expression of PTPRD was observed in UCEC, LUAD, clear cell renal cell carcinoma (ccRCC), ovarian cancer, and breast cancer compared with adjacent normal tissues (Fig. 1D).

\section{Survival analysis}

To investigate the prognostic value of PTPRD across multiple cancers from the TCGA project, the impact of PTPRD expression on survival was evaluated using the GEPIA2 database. As shown in Fig. 2A, low PTPRD expression was associated with a better OS (overall survival) for STAD, uveal melanoma (UVM), SKCM, and BLCA, while low PTPRD expression was related to the poor OS for READ and brain lowergrade glioma (LGG). Besides, low PTPRD expression was linked to a better DFS (disease-free survival) for UVM and KIRP, while low PTPRD expression was related to poor DFS for KIRP. To further investigate the prognostic potential of PTPRD in different tumors, the PrognoScan database was used to assess the relationship between PTPRD expression and prognosis of patients with different cancers based on the data retrieved from Gene Expression Omnibus (GEO) datasets (Supplementary Table 3). The results exhibited that PTPRD expression affected the survival of breast cancer, ovarian cancer, lung cancer, and liposarcoma. As shown in Fig. 2B, the cohort (GSE8894, $N=138, P=0.029)$ of lung cancer patients with low PTPRD expression exhibited a poorer relapse-free survival. Besides, the cohort (GSE30929, N =140, P $=0.003$ ) containing 140 samples of liposarcoma showed that low PTPRD expression was observably associated with improved distant recurrence-free survival (Fig. 2C). Similarly, the GSE26712 (N=185, P = 0.002 ) and GSE9891 ( $\mathrm{N}=278, \mathrm{P}=0.001)$ cohorts containing 185 and 278 ovarian cancer cases, respectively, showed that low PTPRD expression was significantly associated with improved overall survival (Fig. 2D and Fig. 2E). Additionally, patients with low PTPRD expression had poorer disease- 
specific survival in the GSE3494-GPL96 cohort ( $N=236, \mathrm{P}=0.003)$ (Fig. 2F), and patients with low PTPRD expression was correlated with improved distant metastasis-free survival in the GSE2034 cohort $(\mathrm{N}=286, \mathrm{P}=0.030)$ (Fig. 2G). Therefore, it is conceivable that PTPRD expression has an impact on the prognosis of multiple cancers.

\section{Genetic alteration analysis}

It has been broadly recognized that genomic alteration is closely linked to tumorigenesis. To investigate the genomic aberration of PTPRD in pan-cancer, a combined study containing 10967 samples from 32 studies was extracted from the TCGA database for the genetic alteration analysis (Supplementary Table 4). The mutation frequency and genetic alteration type of PTPRD were explored in various cancers (Fig. 3A). The mutation frequency of PTPRD was 9.5\% (1045/10950) in the TCGA pan-cancer cohort with stomach adenocarcinoma (25.2\%) ranking first followed by skin cutaneous melanoma (25.0\%), lung adenocarcinoma (23.1\%), and head and neck squamous cell carcinoma (19.1\%). Besides, the genetic alteration profiling of PTPRD exhibited that its deep deletion was one of the most significant factors for alteration in stomach adenocarcinoma, cholangiocarcinoma, testicular germ cell tumors, head and neck squamous cell carcinoma, lung squamous cell carcinoma, bladder urothelial carcinoma, and lung adenocarcinoma. As shown in Fig. 3B, PTPRD mutations have abundant types of mutation alteration, including missense, nonsense, silent mutation, insertion or deletion, duplication, and frameshift mutation, and G203E, S431L, R705Q, and L1053I sites were observed many times. Moreover, the association between genetic aberrations of PTPRD and the survival prognosis of cases with various types of tumors has been investigated, and the results exhibited that kidney renal clear cell carcinoma cases with altered PTPRD mutations had worse prognosis in disease-specific $(P=1.780 \mathrm{e}-5)$, progression-free $(P=3.899 \mathrm{e}-4)$ and overall $(P=6.366 \mathrm{e}-4)$ survival, but not disease-free $(P=0.595)$ survival, compared with cases with unaltered PTPRD (Fig. 3C, 3D, 3E).

\section{The relationship between PTPRD mutation and response to immunotherapy in pan-cancer}

A combined cohort of 2185 immune checkpoint blockade (ICB)-treated patients (249 from Dana Farber Cancer Institute (DFCl) and 1936 from Memorial Sloan Kettering Cancer Center (MSKCC)) were further used to investigate the relationship between PTPRD mutation and response to ICB therapy (Supplementary Table 5). A comparatively high proportion of PTPRD mutation cases occurred in patients with melanoma (20.59\% of 471 cases) and non-small cell lung cancer ( $12.48 \%$ of 681 cases) (Fig. 4A). Moreover, a significant difference in the number of coexisting mutations between patients with PTPRD mutation type and PTPRD wild type. For instance, PTPRD mutation coexisted more mutated NF1 (36.84\% VS. $8.89 \%)$, PTPRT (34.65\% VS. 8.02\%), GRIN2A (28.95 VS. 5.88\%), PAK5 (25.55\% VS. $4.70 \%$ ) and FLT1 (21.49\% VS. 3.88\%) (Fig. 4B). Besides, TP53, NF1, PTPRT, TERT, PREX2, KMT2D, GRIN2A, KMT2C, FAT1, PAK5 mutations are high-frequency mutations that occur in both PTPRD mutation and PTPRD wild group (Fig. 4C). 
In the cohort of ICB-treated non-small cell lung cancer $(\mathrm{N}=406)$, the OS of the PTPRD-mutant patients ( $N$ $=49$ ) was better than that of those without the PTPRD mutation $(P=0.011)$ (Fig. 4D). Significant differences were found in the distribution of TMB Score $(N=350, P<10 \mathrm{e}-10)$, mutation count $(N=673, P$ $<10 \mathrm{e}-10)$, mutation rate $(\mathrm{N}=240, \mathrm{P}=3.78 \mathrm{e}-10)$, smoker $(\mathrm{N}=296, \mathrm{P}=6.74 \mathrm{e}-8)$, and RECIST $(\mathrm{N}=56, \mathrm{P}=$ 0.002) between the PTPRD-mutation and PTPRD-wildtype groups in the ICB-treated non-small cell lung cancer cohort (Fig. 4E). The ICB-treated non-small cell lung cancer patients with PTPRD mutation have higher TMB scores, more mutation counts, and increased mutation rate. Moreover, the proportion of former smokers (10.26\% VS. 9.73\%), ever smokers (82.05\% VS. $62.65 \%$ ) and current smokers (5.13\% VS. $4.67 \%$ ) in ICB-treated non-small cell lung cancer patients with PTPRD mutation is higher than that of patients without PTPRD mutation (Fig. 4F). Of the six non-small cell lung cancer patients with PTPRD mutation who received immunotherapy, one patient had a complete response (CR), four patients achieved partial response (PR), and one obtained stable disease (SD). Among the 50 non-small cell lung cancer patients without PTPRD mutation who received immunotherapy, 12 patients $(24 \%)$ achieved partial response (PR) and 20 patients (40\%) had stable disease (SD) (Fig. 4G).

For the melanoma patients $(N=471)$ who received immune checkpoint inhibitors, better OS could be observed in the PTPRD-mutation group $(\mathrm{N}=97, \mathrm{P}=0.005)(\mathrm{Fig} .4 \mathrm{H})$. In a subgroup of 471 melanoma patients from the ICB-treated cohort, patients with PTPRD mutation had more mutation counts, and TMB score was significantly higher in PTPRD-mutated patients compared with that in PTPRD-wildtype patients (Fig. 4I).

\section{Immune infiltration analysis}

Immune infiltration was closely associated with the occurrence and development of cancer. Therefore, the relationship between PTPRD expression and the level of immune cell infiltration in various cancers of TCGA was evaluated by investigating the coefficient of PTPRD expression and immune infiltration level using the TIMER database. The findings revealed that PTPRD expression had significant correlations with tumor purity in multiple cancers, including UVM, LUAD, and LUSC. In UVM, the PTPRD expression level was negatively correlated with neutrophil $(r=-0.263, P=0.021)$, and positively correlated with CD8 $+T$ cells $(r=0.251, P=0.028)$ (Fig. 5A). In LUAD, PTPRD expression was positively correlated with CD $4+T$ cells $(r$ $=0.256, P=1.16 \mathrm{e}-08)$, macrophages $(r=0.362, P=1.86 \mathrm{e}-16)$, neutrophils $(r=0.287, P=1.30 \mathrm{e}-10)$, and dendritic cells $(r=0.302, P=9.47 e-12)$ (Fig. 5B). In LUSC, PTPRD expression was positively correlated with macrophages $(r=0.174, P=1.28 \mathrm{e}-04)$ (Fig. $5 \mathrm{C})$. Moreover, we assessed the relationship between PTPRD mutation and immune cell infiltration level in various cancers of TCGA using the TIMER database, and the results showed that the mutated PTPRD status was correlated with high infiltrating levels of CD4 $+T$ cells $(P=0.026)$ in SKCM (Fig. 5D). Additionally, the TISIDB database was used to further evaluate the abundance of tumor-infiltrating lymphocytes (TILs) and PTPRD mutation, and the results showed that PTPRD mutation was positively correlated with memory B cell abundance $(P=0.015)$, and negatively correlated with plasmacytoid dendritic cell $(P=0.009)$ in LUAD (Fig. 5E). Therefore, the findings revealed that PTPRD was closely linked with immune infiltration.

\section{Protein-protein interaction network analysis}


To further investigate the potential mechanisms of PTPRD, we conducted a PPI network of PTPRD based on the top 50 PTPRD-related genes with the STRING database (Supplementary Table 6). 51 nodes and 227 edges were obtained in the PPI network (avg. local clustering coefficient: 0.801, PPI enrichment pvalue: < 1.0e-16) and, the combined score of SLITRK1, IL1RAPL1, SLITRK2, IRS1, and PPFIA1 with PTPRD is $>0.5$ in the PPI network (Fig. 6A). Based on the GEPIA2 database, the expression levels of IRS1 were negatively correlated with PTPRD, and the expression levels of SLITRK1, IL1RAPL1, SLITRK2, and PPFIA1 were positively correlated with PTPRD (Fig. 6B). The findings indicated that SLITRK1, IL1RAPL1, SLITRK2, IRS1, and PPFIA1 were closely related to the modulation and function of PTPRD.

\section{Functional enrichment analysis}

To further explore the underlying mechanism of PTPRD in tumorigenesis, we identified the targeting PTPRD-binding proteins and the PTPRD expression-correlated genes for functional enrichment analysis. We gathered a total of 50 PTPRD-binding proteins using the STRING database, which was validated by experimental evidence (Fig. 6A). Moreover, we used the GEPIA2 database to integrate all TCGA tumor expression data and obtained the top 100 genes related to PTPRD expression (Supplementary Table 7). As shown in Fig. 7A, PTPRD were significantly positively correlated with GATS (GATS, stromal antigen 3 opposite strand) $(R=0.58, P<0.001)$, NRXN1 (neurexin 1) $(R=0.58, P<0.001), Q K I(Q K I, K H$ domain containing, RNA binding) $(R=0.59, P<0.001)$, RP11 (re-mRNA processing factor 31$)(R=0.58, P<0.001)$, and AS1 (prostaglandin D2 receptor (DP) $(R=0.75, P<0.001)$. The top 50 PTPRD-binding proteins were obtained from the PTPRD -target network which was constructed by the STRING database, and the top 100 genes related to PTPRD were screened from the GEPIA2 database. As shown in Fig. 7B, the overlapping target related to PTPRD was SLITRK2 (SLIT and NTRK-like family, member 2) by drawing a Venn diagram. The corresponding heatmap data showed a positive correlation between PTPRD and SLITRK2 in various cancers (Fig. 7C). We combined the top 50 PTPRD-binding proteins and the top 100 genes related to PTPRD to perform KEGG and GO enrichment analyses using the metascape database. The GO enrichment analysis suggested that most of these genes are related to the pathways or cellular biology of RNA metabolisms, such as neuron projection morphogenesis, synapse organization, presynapse, axon, positive regulation of nervous system development, and others (Fig. 7D). The KEGG enrichment analysis indicated that cell adhesion molecules, foxo signaling pathway, AGE-RAGE signaling pathway in diabetic complications, Wnt signaling pathway, GABAergic synapse, hedgehog signaling pathway, neurotrophin signaling pathway, and adherens junction might be involved in the effect of PTPRD on tumor pathogenesis (Fig. 7E).

\section{Discussion}

PTPRD has recently been identified as a potential therapeutic target due to the high prevalence of PTPRD alterations across multiple cancers. The levels of PTPRD mRNA expression were dramatically decreased in liver cirrhosis and HCC cases and were typically increased in healthy liver cases [30]. PTPRD developed both deletion and mutation in several malignancies, and PTPRD deactivation was related to many genetic and epigenetic alterations [5]. However, the underlying molecular mechanisms of PTPRD are still 
largely unclear. Our research offers insights into the underlying role of PTPRD in tumor immunology and its function as a tumor biomarker. Previous studies have illustrated that PTPRD participated in various signaling pathways, including PTPRD/STAT3/JAK, PTPRD/Wnt/ $\beta$-catenin/TCF, PTPRD-CXCL8 axis, PTPRD/PI3K/Akt/mTOR, PTPRD/PD-1/PD-L1 axis [6, 31-33]. Consistent with these findings, our study revealed that PTPRD might modulate cancer-related signaling pathways, such as foxo signaling pathway, Wnt signaling pathway, Hedgehog signaling pathway, and neurotrophin signaling pathway. Therefore, PTPRD is a hopeful therapeutic target across various cancers.

PTPRD, which was involved in the development of glioblastoma multiforme as well as several cancers, could serve as a tumor suppressor. PTPRD suppression appeared in over $50 \%$ glioblastoma multiforme tumors, and reduced expression of PTPRD demonstrated poor prognosis in patients with glioma [5]. Besides, PTPRD mutation was associated with STAT3 activation in HNSCC. However, mRNA expression levels of PTPRD were not related to STAT3 overactivation in HNSCC, suggesting that PTPRD mutation, but not hypermethylation or gene copy number alterations, might be used as a predictive biomarker of sensitivity to STAT3 inhibitors in HNSCC [34]. Furthermore, PTPRD deletion was observed to be associated with a worse prognosis in patients with gastric cancer. Silencing PTPRD remarkably facilitated the proliferation, invasion, and migration of gastric cancer cells via phosphorylating STAT3, suggesting that silencing PTPRD might be an underlying therapeutic target in gastric cancer [35]. Also, heterozygous loss of PTPRD cooperated with Cdkn2a deletion to induce tumorigenesis in glioblastoma. The expression of chemokines, such as CCL2, CCL6, CCL12, and CXCL14, and the polarization of M2 macrophages increased in PTPRD heterozygous tumor cells, indicating that heterozygous PTPRD loss triggered immune activities and affected the macrophage response [36]. These results showed that the function of PTPRD was varied in various types of cancer.

STAT3 has recently appeared as an attractive therapeutic target for various cancers due to its vital role in carcinogenesis. Numerous signaling molecules were involved in STAT3 activation, including ligand binding to growth factor receptors, G-protein coupled receptors (GPCRs), cytokine receptors, toll-like receptors (TLRs), and non-receptor tyrosine kinases. Moreover, IL- 6 stimulated PTPRD by hindering miR34a to suppress overactivation of IL-6/STAT3 signaling in breast cancer [37]. Additionally, STAT3 has also been displayed to bind to the PD-L1 promoter for transcriptional modulation of PD-L1 expression, which can facilitate tumor immune evasion. PTPRD was the phosphatase of STAT3, and loss of PTPRD contributed to the accumulation of phosphorylated STAT3 and STAT3 hyperactivation, which might be linked with response to ICls [36, 38].

It was the first time to investigate PTPRD mutation in pan-cancer and we observed that the proportion of PTPRD mutation was over $15 \%$ in patients with stomach adenocarcinoma, skin cutaneous melanoma, lung adenocarcinoma, head and neck squamous cell carcinoma, and uterine corpus endometrial carcinoma. Moreover, the deep deletion variations of PTPRD were widespread in head and neck squamous cell carcinoma, lung squamous cell carcinoma, bladder urothelial carcinoma, lung adenocarcinoma, and other cancers, which was consistent with the tumor suppressor role of PTPRD in multi-cancer cancers $[6,35]$. Notably, the relationship between PTPRD mutation and response to 
immunotherapy in pan-cancer was investigated for the first time, and the results showed that the OS of the PTPRD-mutant patients was better than that of those without the PTPRD mutation in the cohort of ICB-treated non-small cell lung cancer and melanoma. Previous studies have proved that TMB score could be applied as prognostic indicators for immunotherapy $[39,40]$. We observed that non-small cell lung cancer and melanoma patients with PTPRD mutation had higher TMB scores, consistent with patients with PTPRD mutation or higher TMB scores were more likely to benefit from immunotherapy. Studies in lung cancer patients have demonstrated a stronger response to PD-1 inhibitors in smokers than in non-smokers [41]. Consistently, PTPRD mutations accounted for a higher proportion of lung cancer smokers in our study, indicating that these patients were more likely to benefit from immunotherapy. A further prospective study is warranted to affirm the predictive role of PTPRD in immunotherapy.

To our knowledge, this research was the first study to assess the role of PTPRD mutation in pan-cancer and to examine the relationship between PTPRD and immunotherapy. Our analysis found that PTPRD mutation patients might be more likely to benefit from immune checkpoint inhibitors, especially in nonsmall cell lung cancer and melanoma. Mutations in PTPRD have the potential to be specific immunosubtypes in patients with non-small cell lung cancer and melanoma. Additionally, PTPRD may perform a crucial function in tumorigenesis as a promising molecular target, thus strengthening the comprehension of immunopathogenesis and facilitating the likelihood of discovery and development of new targeted therapeutics.

\section{Declarations}

\section{Funding}

This present study was supported in part by the National Natural Science Foundation of China [grant numbers: 81660755], and the Science and Technology Project of Shenzhen of China [grant numbers: JCYJ20170307160524377 and JCYJ20190808162605484].

\section{Conflicts of interest}

All authors declare that they have no competing interests.

\section{Availability of data and materials}

The data generated or analyzed during this study are included in this article, or if absent are available from the corresponding author upon reasonable request.

\section{Code availability}

Not applicable.

\section{Authors' Contributions}


CPO, QP, and CCZ designed the study and supervised; CPO and QP collected data; CPO performed statistical analysis; CPO interpreted data and drafted the manuscript. CCZ contributed to administrative, technical and material support; all authors reviewed the manuscript and approved the final version.

\section{Ethics approval}

Not applicable.

\section{Consent to participate}

Not applicable.

\section{Consent for publication}

All authors consent to the publication of this research.

\section{Acknowledgments}

We are grateful to the TIMER, GEPIA, GEO, TCGA, UALCAN, PrognoScan, cBioPortal, TISIDB, STRING, and Metascape databases for the availability of the data

\section{References}

1. Siegel RL, Miller KD, Fuchs HE and Jemal A. Cancer Statistics, 2021. CA Cancer J Clin. 2021; 71(1):733.

2. Krzyszczyk P, Acevedo A, Davidoff EJ, Timmins LM, Marrero-Berrios I, Patel M, White C, Lowe C, Sherba JJ, Hartmanshenn C, O'Neill KM, Balter ML, Fritz ZR, et al. The growing role of precision and personalized medicine for cancer treatment. Technology (Singap World Sci). 2018; 6(3-4):79-100.

3. Cancer Genome Atlas Research N, Weinstein JN, Collisson EA, Mills GB, Shaw KR, Ozenberger BA, Ellrott K, Shmulevich I, Sander C and Stuart JM. The Cancer Genome Atlas Pan-Cancer analysis project. Nat Genet. 2013; 45(10):1113-1120.

4. Liu J, Lichtenberg T, Hoadley KA, Poisson LM, Lazar AJ, Cherniack AD, Kovatich AJ, Benz CC, Levine DA, Lee AV, Omberg L, Wolf DM, Shriver CD, et al. An Integrated TCGA Pan-Cancer Clinical Data Resource to Drive High-Quality Survival Outcome Analytics. Cell. 2018; 173(2):400-416 e411.

5. Veeriah S, Brennan C, Meng S, Singh B, Fagin JA, Solit DB, Paty PB, Rohle D, Vivanco I, Chmielecki J, Pao W, Ladanyi M, Gerald WL, et al. The tyrosine phosphatase PTPRD is a tumor suppressor that is frequently inactivated and mutated in glioblastoma and other human cancers. Proc Natl Acad Sci U S A. 2009; 106(23):9435-9440.

6. Huang X, Qin F, Meng Q and Dong M. Protein tyrosine phosphatase receptor type D (PTPRD)mediated signaling pathways for the potential treatment of hepatocellular carcinoma: a narrative review. Ann Transl Med. 2020; 8(18):1192. 
7. Uhl GR and Martinez MJ. PTPRD: neurobiology, genetics, and initial pharmacology of a pleiotropic contributor to brain phenotypes. Ann N Y Acad Sci. 2019; 1451(1):112-129.

8. Funato $\mathrm{K}$, Yamazumi Y, Oda T and Akiyama T. Tyrosine phosphatase PTPRD suppresses colon cancer cell migration in coordination with CD44. Exp Ther Med. 2011; 2(3):457-463.

9. Bae WJ, Ahn JM, Byeon HE, Kim S and Lee D. PTPRD-inactivation-induced CXCL8 promotes angiogenesis and metastasis in gastric cancer and is inhibited by metformin. $\mathrm{J}$ Exp Clin Cancer Res. 2019; 38(1):484.

10. Onoi K, Chihara Y, Uchino J, Shimamoto T, Morimoto Y, Iwasaku M, Kaneko Y, Yamada T and Takayama K. Immune Checkpoint Inhibitors for Lung Cancer Treatment: A Review. J Clin Med. 2020; 9(5).

11. Goodman AM, Sokol ES, Frampton GM, Lippman SM and Kurzrock R. Microsatellite-Stable Tumors with High Mutational Burden Benefit from Immunotherapy. Cancer Immunol Res. 2019; 7(10):15701573.

12. Sha D, Jin Z, Budczies J, Kluck K, Stenzinger A and Sinicrope FA. Tumor Mutational Burden as a Predictive Biomarker in Solid Tumors. Cancer Discov. 2020; 10(12):1808-1825.

13. Lu Z, Chen H, Jiao X, Zhou W, Han W, Li S, Liu C, Gong J, Li J, Zhang X, Wang X, Peng Z, Qi C, et al. Prediction of immune checkpoint inhibition with immune oncology-related gene expression in gastrointestinal cancer using a machine learning classifier. J Immunother Cancer. 2020; 8(2).

14. Ready N, Hellmann MD, Awad MM, Otterson GA, Gutierrez M, Gainor JF, Borghaei H, Jolivet J, Horn L, Mates M, Brahmer J, Rabinowitz I, Reddy PS, et al. First-Line Nivolumab Plus Ipilimumab in Advanced Non-Small-Cell Lung Cancer (CheckMate 568): Outcomes by Programmed Death Ligand 1 and Tumor Mutational Burden as Biomarkers. J Clin Oncol. 2019; 37(12):992-1000.

15. Solomon DA, Kim JS, Cronin JC, Sibenaller Z, Ryken T, Rosenberg SA, Ressom H, Jean W, Bigner D, Yan $\mathrm{H}$, Samuels $\mathrm{Y}$ and Waldman T. Mutational inactivation of PTPRD in glioblastoma multiforme and malignant melanoma. Cancer Res. 2008; 68(24):10300-10306.

16. Li T, Fu J, Zeng Z, Cohen D, Li J, Chen Q, Li B and Liu XS. TIMER2.0 for analysis of tumor-infiltrating immune cells. Nucleic Acids Res. 2020; 48(W1):W509-W514.

17. Chandrashekar DS, Bashel B, Balasubramanya SAH, Creighton CJ, Ponce-Rodriguez I, Chakravarthi B and Varambally S. UALCAN: A Portal for Facilitating Tumor Subgroup Gene Expression and Survival Analyses. Neoplasia. 2017; 19(8):649-658.

18. Mizuno H, Kitada K, Nakai K and Sarai A. PrognoScan: a new database for meta-analysis of the prognostic value of genes. BMC Med Genomics. 2009; 2:18.

19. Gao J, Aksoy BA, Dogrusoz U, Dresdner G, Gross B, Sumer SO, Sun Y, Jacobsen A, Sinha R, Larsson E, Cerami E, Sander $\mathrm{C}$ and Schultz N. Integrative analysis of complex cancer genomics and clinical profiles using the cBioPortal. Sci Signal. 2013; 6(269):pl1.

20. Samstein RM, Lee CH, Shoushtari AN, Hellmann MD, Shen R, Janjigian YY, Barron DA, Zehir A, Jordan EJ, Omuro A, Kaley TJ, Kendall SM, Motzer RJ, et al. Tumor mutational load predicts survival after immunotherapy across multiple cancer types. Nat Genet. 2019; 51(2):202-206. 
21. Miao D, Margolis CA, Vokes NI, Liu D, Taylor-Weiner A, Wankowicz SM, Adeegbe D, Keliher D, Schilling B, Tracy A, Manos M, Chau NG, Hanna GJ, et al. Genomic correlates of response to immune checkpoint blockade in microsatellite-stable solid tumors. Nat Genet. 2018; 50(9):1271-1281.

22. Rizvi H, Sanchez-Vega F, La K, Chatila W, Jonsson P, Halpenny D, Plodkowski A, Long N, Sauter JL, Rekhtman N, Hollmann T, Schalper KA, Gainor JF, et al. Molecular Determinants of Response to AntiProgrammed Cell Death (PD)-1 and Anti-Programmed Death-Ligand 1 (PD-L1) Blockade in Patients With Non-Small-Cell Lung Cancer Profiled With Targeted Next-Generation Sequencing. J Clin Oncol. 2018; 36(7):633-641.

23. Rizvi NA, Hellmann MD, Snyder A, Kvistborg P, Makarov V, Havel JJ, Lee W, Yuan J, Wong P, Ho TS, Miller ML, Rekhtman N, Moreira AL, et al. Cancer immunology. Mutational landscape determines sensitivity to PD-1 blockade in non-small cell lung cancer. Science. 2015; 348(6230):124-128.

24. Li T, Fan J, Wang B, Traugh N, Chen Q, Liu JS, Li B and Liu XS. TIMER: A Web Server for Comprehensive Analysis of Tumor-Infiltrating Immune Cells. Cancer Res. 2017; 77(21):e108-e110.

25. Li B, Severson E, Pignon JC, Zhao H, Li T, Novak J, Jiang P, Shen H, Aster JC, Rodig S, Signoretti S, Liu JS and Liu XS. Comprehensive analyses of tumor immunity: implications for cancer immunotherapy. Genome Biol. 2016; 17(1):174.

26. Ru B, Wong CN, Tong Y, Zhong JY, Zhong SSW, Wu WC, Chu KC, Wong CY, Lau CY, Chen I, Chan NW and Zhang J. TISIDB: an integrated repository portal for tumor-immune system interactions. Bioinformatics. 2019; 35(20):4200-4202.

27. Tang Z, Li C, Kang B, Gao G, Li C and Zhang Z. GEPIA: a web server for cancer and normal gene expression profiling and interactive analyses. Nucleic Acids Res. 2017; 45(W1):W98-W102.

28. Szklarczyk D, Gable AL, Lyon D, Junge A, Wyder S, Huerta-Cepas J, Simonovic M, Doncheva NT, Morris JH, Bork P, Jensen LJ and Mering CV. STRING v11: protein-protein association networks with increased coverage, supporting functional discovery in genome-wide experimental datasets. Nucleic Acids Res. 2019; 47(D1):D607-D613.

29. Zhou Y, Zhou B, Pache L, Chang M, Khodabakhshi AH, Tanaseichuk O, Benner C and Chanda SK. Metascape provides a biologist-oriented resource for the analysis of systems-level datasets. Nat Commun. 2019; 10(1):1523.

30. Acun T, Demir K, Oztas E, Arango D and Yakicier MC. PTPRD is homozygously deleted and epigenetically downregulated in human hepatocellular carcinomas. OMICS. 2015; 19(4):220-229.

31. Tomita H, Cornejo F, Aranda-Pino B, Woodard CL, Rioseco CC, Neel BG, Alvarez AR, Kaplan DR, Miller FD and Cancino GI. The Protein Tyrosine Phosphatase Receptor Delta Regulates Developmental Neurogenesis. Cell Rep. 2020; 30(1):215-228 e215.

32. Hsu HC, Lapke N, Chen SJ, Lu YJ, Jhou RS, Yeh CY, Tsai WS, Hung HY, Hsieh JC, Yang TS, Thiam TK and You JF. PTPRT and PTPRD Deleterious Mutations and Deletion Predict Bevacizumab Resistance in Metastatic Colorectal Cancer Patients. Cancers (Basel). 2018; 10(9).

33. UhI GR, Martinez MJ, Paik P, Sulima A, Bi GH, lyer MR, Gardner E, Rice KC and Xi ZX. Cocaine reward is reduced by decreased expression of receptor-type protein tyrosine phosphatase $D$ (PTPRD) and by 
a novel PTPRD antagonist. Proc Natl Acad Sci U S A. 2018; 115(45):11597-11602.

34. Peyser ND, Du Y, Li H, Lui V, Xiao X, Chan TA and Grandis JR. Loss-of-Function PTPRD Mutations Lead to Increased STAT3 Activation and Sensitivity to STAT3 Inhibition in Head and Neck Cancer. PLoS One. 2015; 10(8):e0135750.

35. Wu L, Gao L, Kong D and Xue H. Loss of Tyrosine Phosphatase Delta Promotes Gastric Cancer Progression via Signal Transducer and Activator of Transcription 3 Pathways. Dig Dis Sci. 2019; 64(11):3164-3172.

36. Ortiz B, Fabius AW, Wu WH, Pedraza A, Brennan CW, Schultz N, Pitter KL, Bromberg JF, Huse JT, Holland EC and Chan TA. Loss of the tyrosine phosphatase PTPRD leads to aberrant STAT3 activation and promotes gliomagenesis. Proc Natl Acad Sci U S A. 2014; 111(22):8149-8154.

37. Zhang F, Wang B, Qin T, Wang L, Zhang Q, Lu Y, Song B, Yu X and Li L. IL-6 induces tumor suppressor protein tyrosine phosphatase receptor type $D$ by inhibiting miR-34a to prevent IL-6 signaling overactivation. Mol Cell Biochem. 2020; 473(1-2):1-13.

38. Ding L, Chen X, Xu X, Qian Y, Liang G, Yao F, Yao Z, Wu H, Zhang J, He Q and Yang B. PARP1 Suppresses the Transcription of PD-L1 by Poly(ADP-Ribosyl)ating STAT3. Cancer Immunol Res. 2019; 7(1):136-149.

39. Mo J, Hu X, Gu L, Chen B, Khadaroo PA, Shen Z, Dong L, Lv Y, Chitumba MN and Liu J. Smokers or non-smokers: who benefits more from immune checkpoint inhibitors in treatment of malignancies? An up-to-date meta-analysis. World J Surg Oncol. 2020; 18(1):15.

40. Gibney GT, Weiner LM and Atkins MB. Predictive biomarkers for checkpoint inhibitor-based immunotherapy. Lancet Oncol. 2016; 17(12):e542-e551.

41. Norum $\mathrm{J}$ and Nieder $\mathrm{C}$. Tobacco smoking and cessation and PD-L1 inhibitors in non-small cell lung cancer (NSCLC): a review of the literature. ESMO Open. 2018; 3(6):e000406.

\section{Figures}




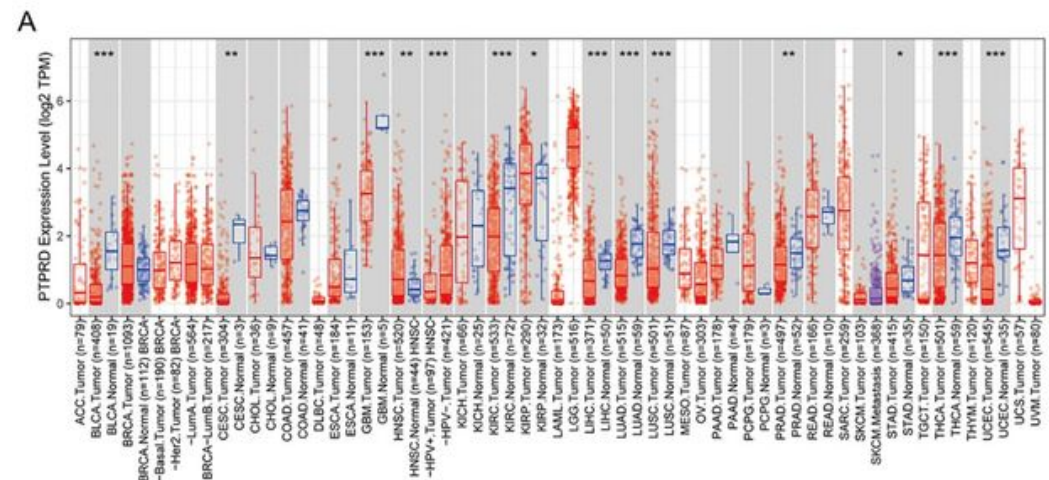

B
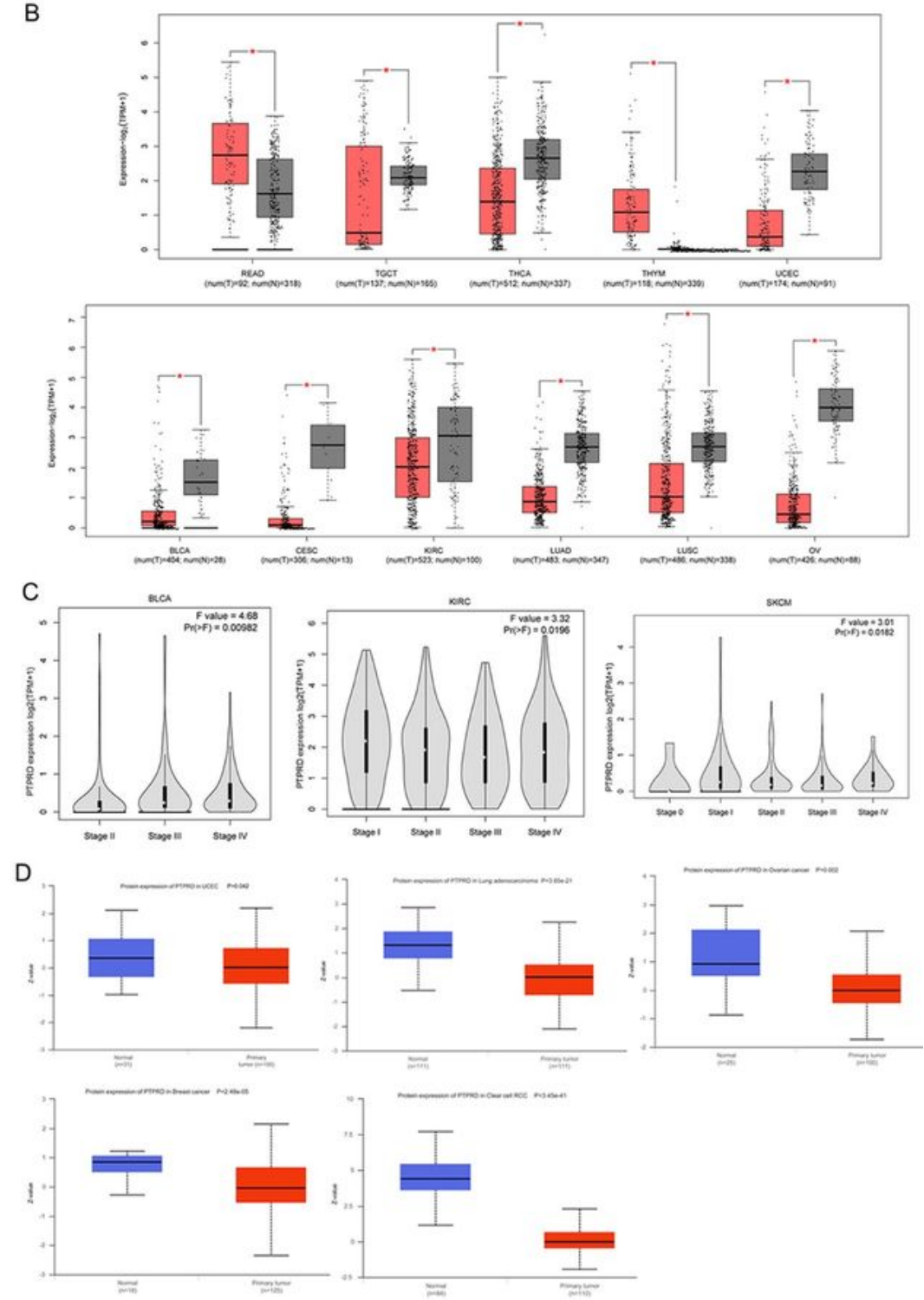

Figure 1

PTPRD expression level in various tumors. (a) PTPRD expression was assessed across 23 cancer types from the TCGA project using the TIMER database. * $P<0.05$; ${ }^{\star \star} P<0.01$; $* \star \star ~ P<0.001$. (b) The box plot of PTPRD expression was explored between cancer tissues and normal tissues across 33 cancer types from TCGA and GTEx projects using the GEPIA2 database. $* \star ~ P<0.01$. (c) The relationship between PTPRD expression and pathological stages of BLCA, KIRC, and SKCM from TCGA project using GEPIA2 
database. Log2 (TPM+1) was applied for log-scale. (d) The expression level of PTPRD total protein was investigated between normal tissue and primary tissue of breast cancer, LUAD, ovarian cancer, CcRCC, and UCEC using the CPTAC dataset. *** $\mathrm{P}<0.001$.

A
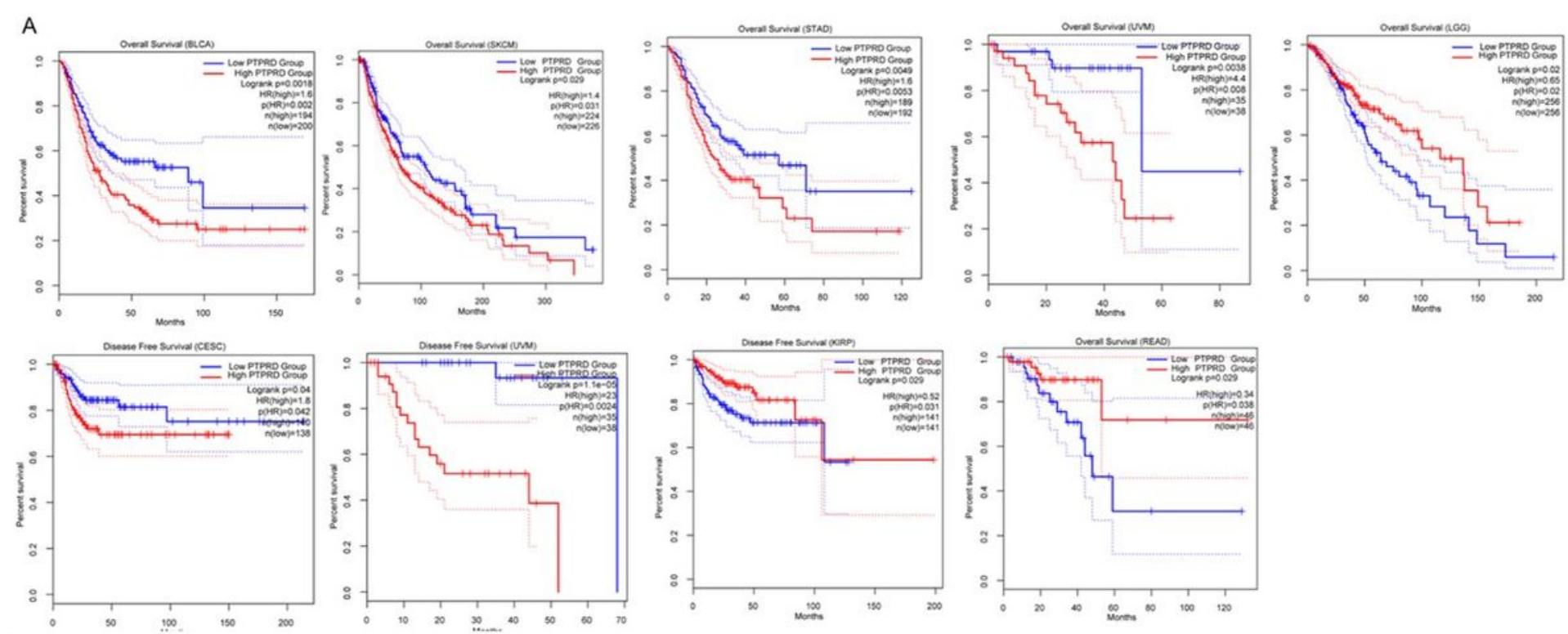

B
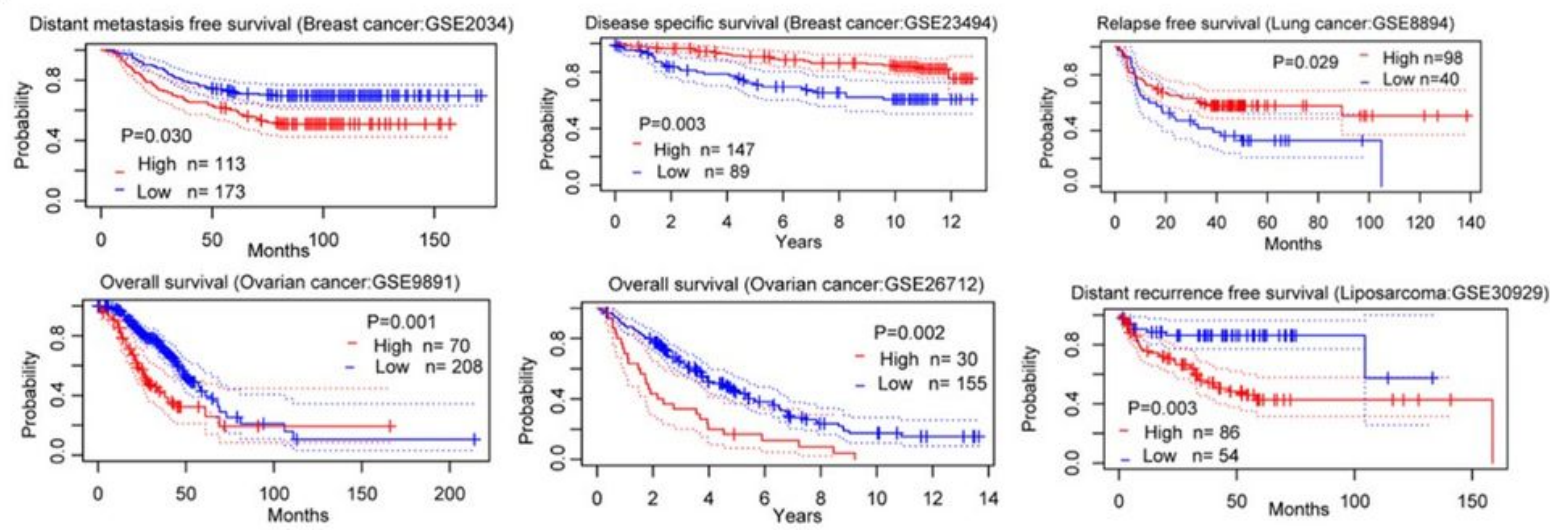

\section{Figure 2}

Relationship between PTPRD expression and survival in various cancers (a) The TCGA data from the GEPIA2 database was used to assess the impact of PRPRD expression on OS (overall survival) and DFS (disease-free survival). (b) The PrognoScan database was used to assess the relationship between PTPRD expression and survival based on the data retrieved from GEO datasets. 

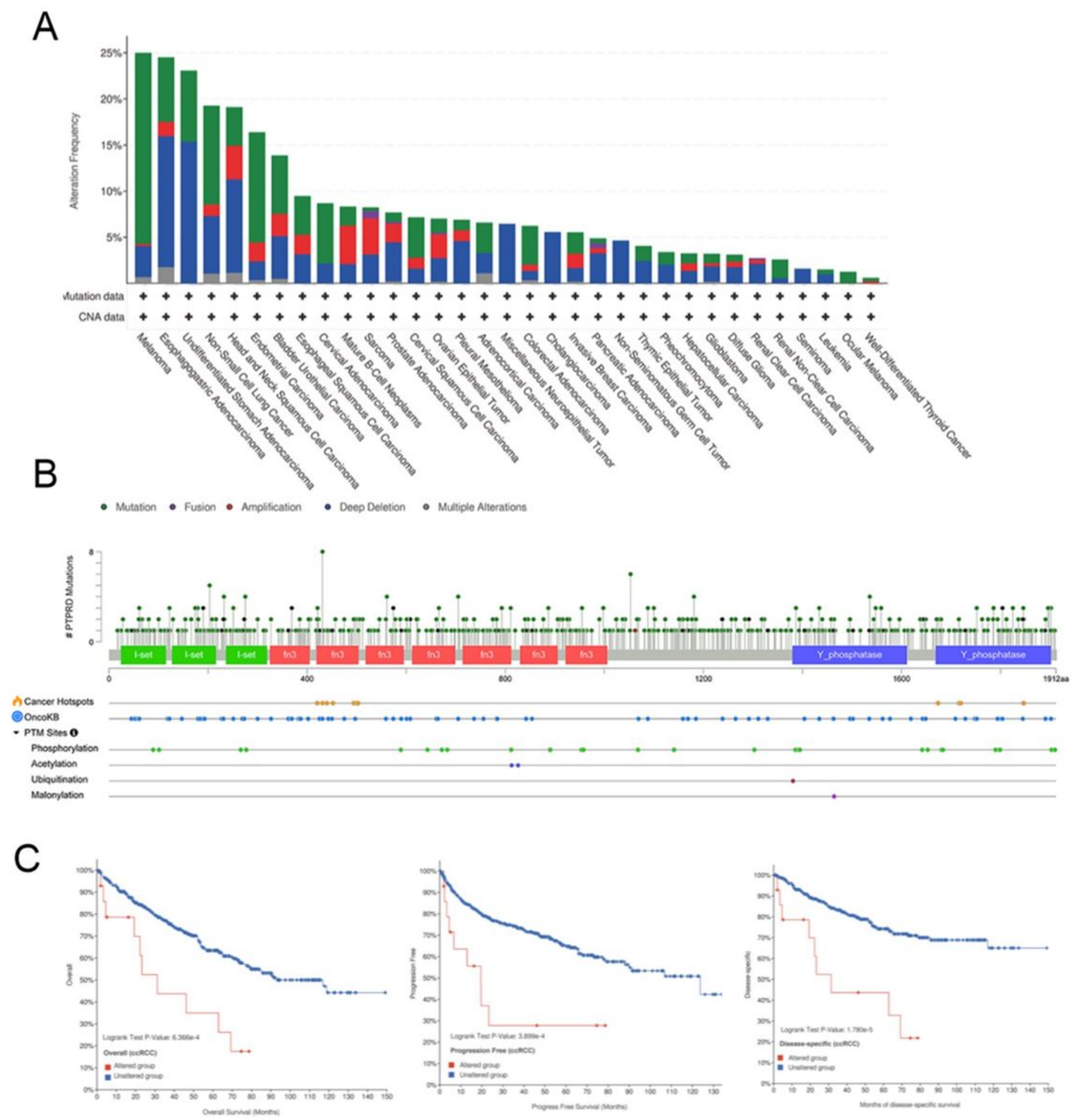

Figure 3

Genetic alteration of PTPRD in various cancers of TCGA using the cBioPortal database. (a) The alteration frequency and type of PTPRD in pan-cancer. (b) The alteration sites and types of PRPRD in pan-cancer. (c) The correlation between PRPRD mutation and overall, disease-specific, and progression-free survival of ccRCC. A combined study containing 10967 samples from 32 studies was extracted from the TCGA database. 

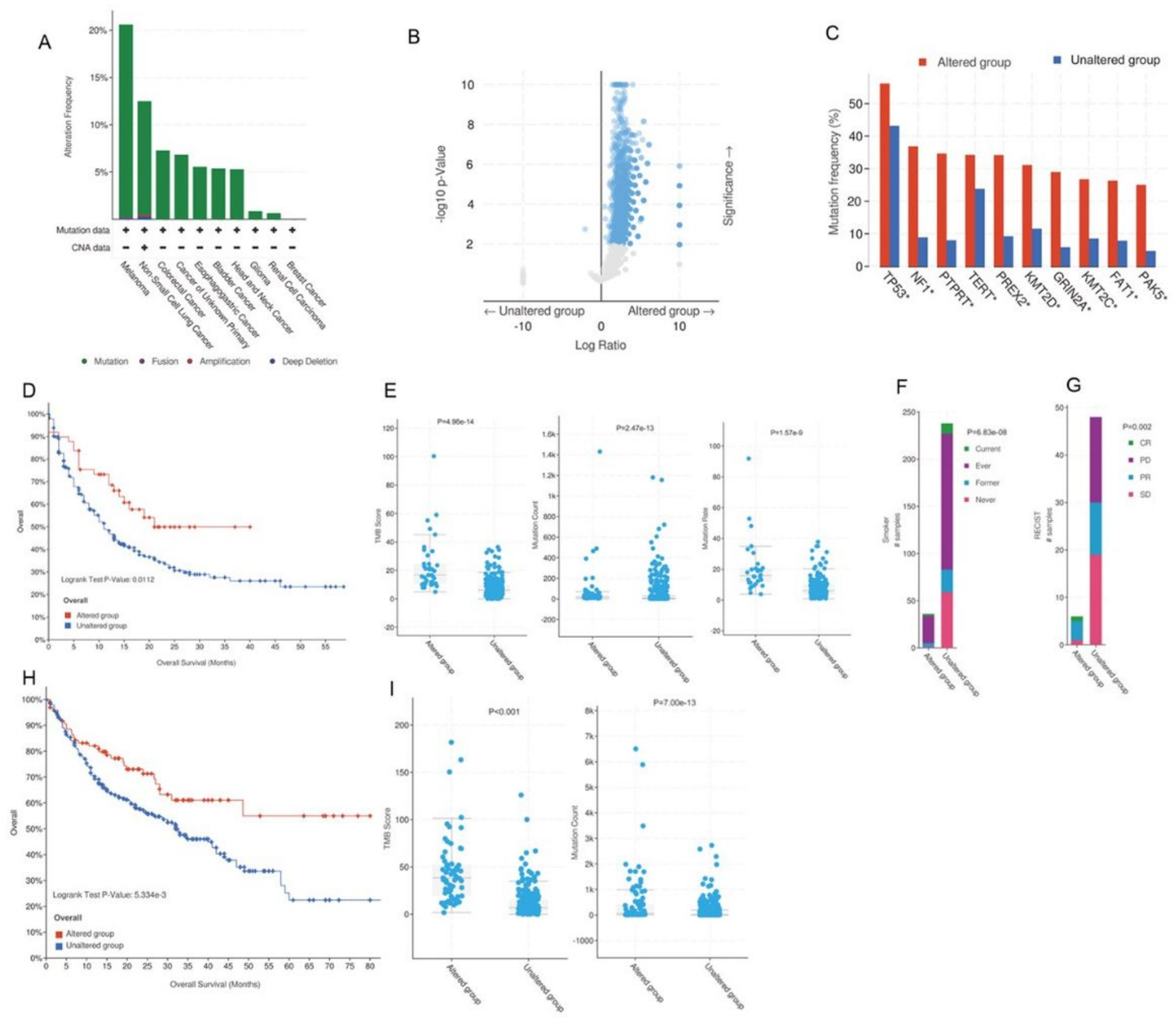

\section{Figure 4}

The relationship between PTPRD mutation and response to immunotherapy in pan-cancer using the cBioPortal database. (a) The proportion of PRPRD mutation cases occurred in pan-cancer. (b) Coexisting mutations between patients with PTPRD or not in pan-cancer. (c) High-frequency mutations occurred in both the PTPRD mutation and PTPRD wild group. (d) The OS of PTPRD-mutation and PTPRD-wildtype groups in ICB-treated non-small cell lung cancer cohort. (e) The relationship between PTPRD mutation and TMB score, mutation count, and mutation rate in ICB-treated non-small cell lung cancer cohort. (f) The relationship between PTPRD mutation and smoke in ICB-treated non-small cell lung cancer cohort. (g) The relationship between PTPRD mutation and RECIST (Response Evaluation Criteria) in ICB-treated non-small cell lung cancer cohort. (h) The OS of PTPRD-mutation and PTPRD-wildtype groups in ICBtreated melanoma cohort. (i) The relationship between PTPRD mutation and TMB score and mutation 
count in ICB-treated melanoma cohort. A combined study containing 2185 patients who received ICBs therapy was extracted from four studies of the TCGA database.
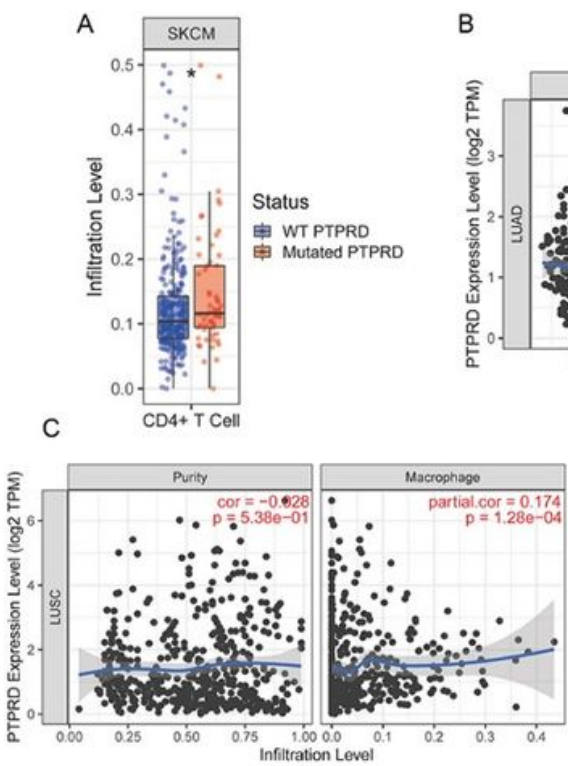

B

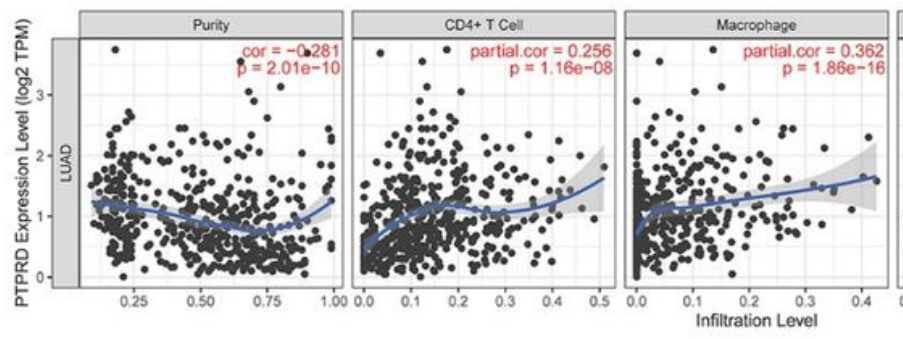

$\mathrm{D}$

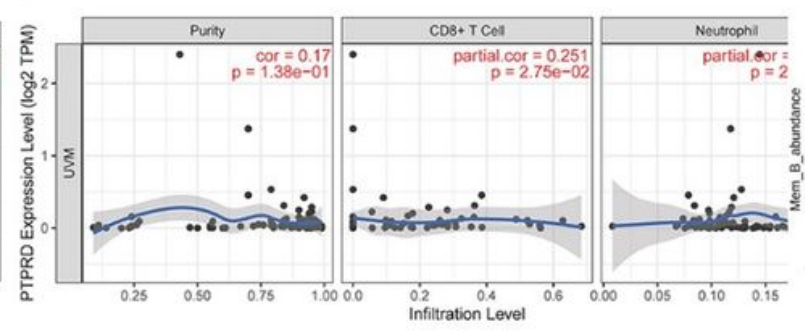

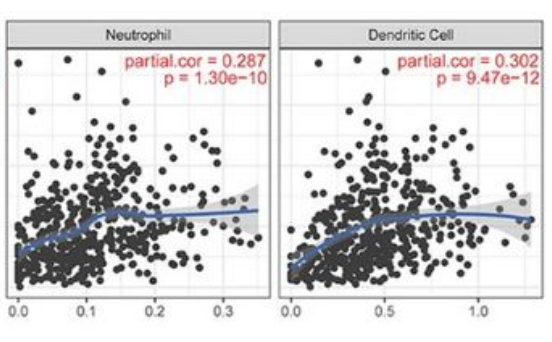

$E$
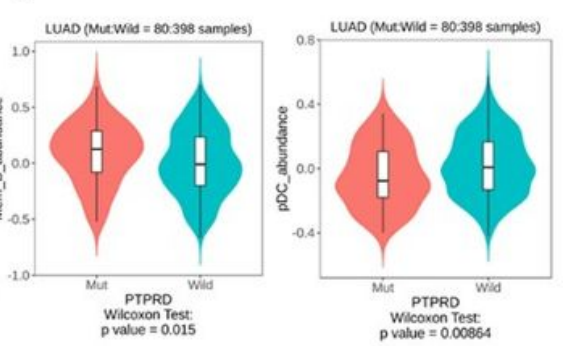

Figure 5

Correlation between PTPRD and immune infiltration. (a) The correlation between PTPRD expression and immune infiltration was explored in UVM using the TIMER database. (b) The correlation between PTPRD expression and immune infiltration was investigated in LUAD using the TIMER database. (c) The correlation between PTPRD expression and immune infiltration was explored in LUSC using the TIMER database. (d) The relationship between PTPRD mutation and immune infiltration in SKCM using the TIMER database. (e) The relationship between PTPRD mutation and the abundance of tumor-infiltrating lymphocytes (TILs) was assessed using the TISIDB database.
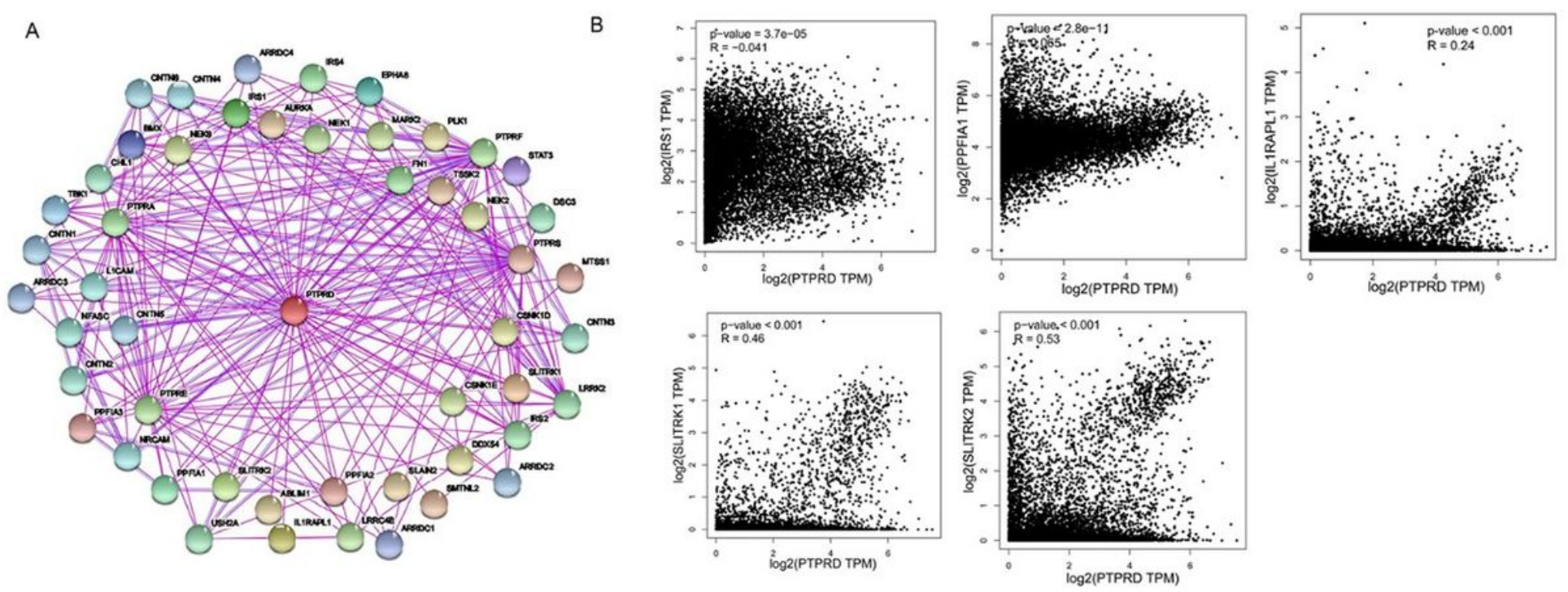

$\log _{2}^{2}$ (PTPŔD TPM)

Figure 6 
Protein-protein interaction network analysis. (a) a protein-protein interaction network was conducted based on the 50 PTPRD-related genes with STRING database. (b) Correlation between PTPRD expression and SLITRK1, IL1RAPL1, SLITRK2, IRS1, and PPFIA1 expression.

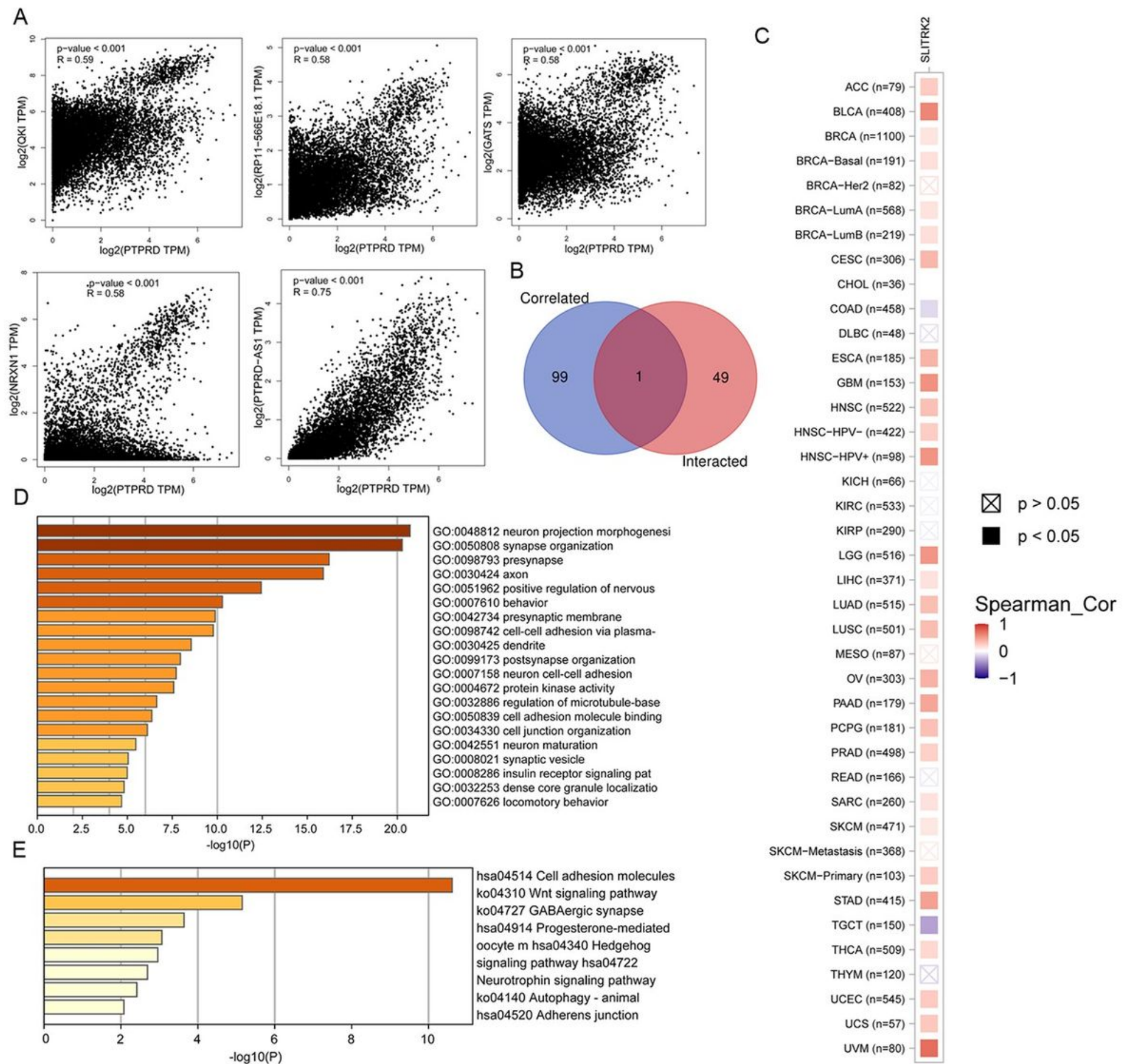

\section{Figure 7}

Functional enrichment analysis. (a) We obtained the top 100 PTPRD-correlated genes in the TCGA project using the GEPIA2 database and investigated the expression correlation between PTPRD and the top five PTPRD-correlated genes, including GATS, NRXN1, QKI, RP11, and AS1. (b) A Venn diagram was drawn using the 50 PTPRD -binding proteins from the STRING database and 100 genes related to PTPRD from the GEPIA2 database. (c) The corresponding heatmap data exhibited the correlation between PTPRD and 
SLITRK2 across various cancers from the TCGA using the GEPIA2 database. (d) Go enrichment analysis was performed using the metascape database. (e) KEGG enrichment analysis was performed using the metascape database.

\section{Supplementary Files}

This is a list of supplementary files associated with this preprint. Click to download.

- SupplementaryData.docx 